\title{
Der Einfluss von ökologischen Produkteigenschaften bei PCs auf die Kaufentscheidung
}

\section{Ergebnisse einer Conjoint-Analyse}

\author{
Timo Schmidtchen', Nils-Holger Schmidt', Lutz M. Kolbe', Jutta Geldermann² \\ ${ }^{1}$ Professur für Informationsmanagement, Georg-August-Universität Göttingen \\ 2 Professur für Produktion und Logistik, Georg-August-Universität Göttingen
}

\section{Einleitung}

Aus der zunehmenden Bedeutung von Informationssystemen für die Wirtschaft resultieren ein steigender Energiebedarf und eine zunehmende Umweltbelastung. Im Jahr 2005 benötigten Server und deren Infrastruktur weltweit 123.000 Gigawattstunden Energie. Von insgesamt 820 Millionen Tonnen $\mathrm{CO}_{2}$-Ausstoß pro Jahr gehen zwei Prozent zu Lasten der IT (Heng 2009, S. 1). Dabei spielt die Diskussion um Green IT eine wesentliche Rolle. (Schmidt et al. 2009, S. 1)

Wissenschaftliche Literatur zur Green IT befasst sich überwiegend mit der Situation in Unternehmen. Entscheidend für ein Umdenken in der Gesellschaft kann nicht ausschließlich die Unternehmensseite sein. Zwangsläufig muss die Frage gestellt werden, ob ökologische Produkteigenschaften einen Einfluss auf die Kaufentscheidung von Konsumenten ausüben. Beispielsweise verfolgt der ITHardwarehersteller Apple mit dem MacBook Pro bereits eine Green-IT-Strategie für Notebooks. Der Bildschirm dieser Produktvariante soll nach Herstellerangaben $23 \%$ weniger Strom im Vergleich zu den anderen Bildschirmen der Produktreihe benötigen und frei von Quecksilber und Arsen sein (Apple Inc. 2009, S. 1-3).

Andere Forschungsarbeiten haben gezeigt, dass nachhaltige Produkte das Konsumentenverhalten positiv beeinflussen können (Du et al. 2007, S. 224-241; Lichtenstein et al. 2004, S. 16-32; Luo und Bhattacharya 2006, S. 1-18). Daraus leitet sich die Frage ab, ob ökologische Produkteigenschaftenauch bei PCs (Personal Computer) einen Einfluss auf das Konsumentenverhalten haben. Im Rahmen dieser Arbeit wurden 500 Personen unterschiedliche PC-Konzepte zur Bildung einer Präferenzreihenfolge vorgelegt. Mittels einer Conjoint-Analyse wurden dann die partiellen Teilnutzenwerte von ökologischen Produkteigenschaftenbei PCs bestimmt und eine Cluster- und Diskriminanzanalyse durchgeführt. Hieraus lassen 
sich Handlungsempfehlungen für die Produktentwicklung und das Marketing von IT-Hardwarehersteller sowie IT-Organisationen ableiten, die umweltorientierte Kundenbedürfnisse zukünftig stärker bedienen wollen.

\section{Grundlagen und Fragestellungen}

Für Green IT finden sich in Praxis und Wissenschaft zahlreiche unterschiedliche Definitionen. Im deutschsprachigen Raum hat sich die Definition des Branchenverbandes BITKOM (Bundesverband Informationswirtschaft, Telekommunikation und neue Medien e.V.) durchgesetzt, der Green IT wie folgt definiert:

„Der Begriff Green IT umfasst [...] die Gesamtheit aller ITK-basierten Potenziale für Energie- und Ressourceneffizienz - und dies über den gesamten Lebenszyklus vom Design zur Produktion über die Nutzung bis hin zur Entsorgung. Dabei steht der Energieeinsatz bzw. die Energieeffizienz in der Gebrauchsphase von ITK-Geräten im Vordergrund aktueller Aktivitäten" (BITKOM 2009b, S. 1).

Green IT umfasst somit den energieeffizienten Gebrauch und die umweltgerechte Entsorgung von ITK (Informations- und Telekommunikationstechnologie), zu der auch PCs gezählt werden.

Erste Untersuchungen haben gezeigt, dass ökologische Produkteigenschaften, operationalisiert durch den Energieverbrauch und die Entsorgungseigenschaften, einen Einfluss auf die Beurteilung von ITK, zum Beispiel Notebooks, Mobiltelefonen und PCs haben (BITKOM 2009a, S. 1). Daraus leitet sich für den spezifischen Fall der PCs die Frage ab, ob Konsumenten ökologische Produkteigenschaftenbei ihrer Kaufentscheidung mit berücksichtigen:

Frage 1: Welchen Einfluss haben ökologische Produkteigenschafteneines PCs auf die Kaufentscheidung der Konsumenten?

Gemäß der Operationalisierung von ökologischen Produkteigenschaften nach BITKOM (2009a, S. 1) in Energie- und Entsorgungseigenschaften folgen daraus zwei abgeleitete Fragestellungen:

Frage 2: Welchen Einfluss haben Energieeigenschaften eines PCs auf die Kaufentscheidung der Konsumenten?

Frage 3: Welchen Einfluss baben Entsorgungseigenschaften eines PCs auf die Kaufentscheidung der Konsumenten?

Im nächsten Schritt stellt sich die Frage, wie groß der potenzielle Markt für PCs mit ökologischen Produkteigenschaften ist. Anhaltspunkte hierfür gibt die Konsumentengruppe der sogenannten „LOHAS”. „LOHAS“ steht für Lifestyle of Health and Sustainability und beschreibt eine „Bewegung mit starkem Einfluss auf Konsum und Werteentwicklung" (Wenzel et al. 2007, S. 6) in der Gesellschaft. Der 
Lebensstil beschreibt keine elitäre Zielgruppe, sondern „eine neue gesellschaftliche Mehrheit" (Wenzel et al. 2007, S. 3). Der Trend in der Lebensmittelbranche hin zu Bio-Produkten wird laut Wenzel et al. (2007, S. 118) weitere Branchen erreichen.

Der wachsende Anteil der LOHAS auf dem deutschen Markt wurde im Jahr 2007 auf ein Drittel geschätzt (Wenzel et al. 2007, S. 111). Werden diese Erkenntnisse auf den Markt für PCs übertragen, so kann vermutet werden, dass ca. ein Drittel der Konsumenten einen PC mit ökologischen Produkteigenschaften einem konventionellen PC vorziehen würde.

Frage 4: Wie groß ist der potenzielle Markt für PCs mit ökologischen Produkteigenschaf-
ten?

\section{Methodik}

Die Conjoint-Analyse wird für die Planung von neuen Produkten und Dienstleistungen verwendet. Eine Conjoint-Analyse beschreibt „empirische Verfahren, welche die Nutzenvorstellungen beziehungsweise Präferenzen von Testpersonen ermitteln“ (Meffert 2000, S. 170). Mithilfe der traditionellen Conjoint-Analyse können Marktanteile mit einer Abweichung von 5,1\% vorhergesagt werden (Heidbrink 2006, S. 110). Es handelt sich um ein multivariates Verfahren der Interdependenzanalyse. Der Conjoint-Analyse wird eine große Bedeutung in der Marktforschung beigemessen (Dellaert et al. 1996, S. 1; Wittink et al. 1994, S. 51). Eine Simulation mittels Conjoint-Analyse ermöglicht es, alternative Produktkonzepte für den Markt zu testen (Wyner 1992, S. 43). Dabei haben die Befragten die Aufgabe eine repräsentative Auswahl von Produktkonzepten in eine der Präferenz nach geordnete Reihenfolge zu bringen. Anhand dieser Gesamturteile lassen sich relative Wichtigkeiten und Teilnutzenwerte der Eigenschaftsausprägungen berechnen. Für die reale Kaufbereitschaft sind durch die Conjoint-Analyse im Vergleich zu einem kompositionellen Verfahren genauere Ergebnisse zu erwarten (Heidbrink 2006, S. 110; Backhaus et al. 2006, S. 558; Diller 2008, S. 190; Foscht und Swoboda 2007, S. 72-73; Görts und Behringer 2003, S. 285-286; Meffert 2000, S. 170; Müller-Hagedorn 2005, S. 115-116). Für die Durchführung einer Conjoint-Analyse empfiehlt sich der Einsatz eines Online-Fragebogens, um Daten schneller, mit einer größeren Stichprobe und zugleich geringeren Kosten erheben zu können (Zerr 2003, S. 13-14).

Für die Erhebung der Daten wurde ein Online-Fragebogen mit zwölf verschieden Produktkonzepte von PCs entwickelt. Drei dieser Produktkonzepte dienten als Prüffälle zur Bestimmung der Validität. Zur Durchführbarkeit der Conjoint-Analyse wurden die PCs durch die vier Eigenschaften Preis, Leistung, Energieverbrauch und Entsorgung mit jeweils drei Eigenschaftsausprägungen beschrieben. 
Die Ergebnisse dieser Untersuchung beschränken sich damit auf diese Eigenschaften und die jeweiligen Eigenschaftsausprägungen. Andere Eigenschaften von PCs, die eine wichtige Rolle hätten spielen können, wurden nicht betrachtet.

Die Eigenschaftsausprägungen orientieren sich an markttypischen Merkmalen von PCs. In der Regel werden Eigenschaften im Rahmen einer Conjoint-Analyse als diskret betrachtet (Albers 1984, S. 311). Daher wurde die Annahme getroffen, dass es sich bei den Eigenschaftsausprägungen für Preis, Leistung, Energieverbrauch und Entsorgungseigenschaften um diskrete Merkmale handelt.

Befragt wurden Internetnutzer, die per Bannerrotation auf ca. 30.000 deutschsprachigen Internetseiten auf den Fragebogen aufmerksam gemacht wurden. Im Zeitraum vom 16. Mai 2009 bis 11. Juli 2009 nahmen 556 Personen an der Umfrage teil. Aufgrund offensichtlicher Falschangaben und nicht logischer Zusammenhänge wurde die Umfrage um 56 Datensätze bereinigt, sodass 500 Datensätze zur weiteren Analyse zur Verfügung standen.

Die Aussagen dieser Studie sind limitiert auf Onlinenutzer aus dem deutschsprachigen Raum. Die Selbstselektion der Teilnehmer bei der Erhebung der Daten war nicht zu umgehen. Daraus ergibt sich, dass möglicherweise Konsumenten, die sich im höheren Maße für PCs interessieren als der Durchschnitt, mit einer höheren Wahrscheinlichkeit an der Umfrage teilgenommen haben.

Neben den Präferenzen bezüglich der verschiedenen Produktkonzepte wurden zusätzlich soziodemographische Faktoren und allgemeine Daten zur Nutzung von PCs erfasst. Die Stichprobe setzt sich zu 80,2\% aus Männern und zu 19,8 \% aus Frauen zusammen. Das Durchschnittsalter liegt bei 26,6 Jahren.

\section{Analyse und Interpretation der Ergebnisse}

Die Bildung einer Präferenzreihenfolge zeigt, welche PCs die erste Präferenz für die Befragten hatten (vgl. Tabelle 1). Bei der Beschreibung der Leistung handelt es sich streng genommen nicht um reale Prozessoren, sondern um einen Prozessor mit mehreren Kernen.

Tabelle 1: Präferenzreihenfolge der PC-Konzepte für die Befragten

\begin{tabular}{|l|l|c|}
\hline Rang & Eigenschaftsausprägungen des PCs & $\begin{array}{c}\text { Erste Präferenz für } \\
\text { die Befragten }\end{array}$ \\
\hline 1. & $\begin{array}{l}\text { Leistung: 4 Prozessoren mit je 3 GHz } \\
\text { Preis: 400 Euro } \\
\text { Energie: Energieverbrauch von 100 Watt } \\
\text { Entsorgung: Keine Information über Recycling }\end{array}$ & $\mathrm{n}=228$ \\
$45,6 \%$ \\
\hline
\end{tabular}




\begin{tabular}{|l|l|c|}
\hline 2. & $\begin{array}{l}\text { Leistung: 4 Prozessoren mit je 3 GHz } \\
\text { Preis: 400 Euro } \\
\text { Energie: Energieverbrauch von 175 Watt } \\
\text { Entsorgung: PC kann komplett recycelt werden }\end{array}$ & $\mathrm{n}=125$ \\
$25,0 \%$ \\
\hline 3. & $\begin{array}{l}\text { Leistung: 2 Prozessoren mit je 3 GHz } \\
\text { Preis: 500 Euro } \\
\text { Energie: Energieverbrauch von 100 Watt } \\
\text { Entsorgung: PC kann komplett recycelt werden }\end{array}$ & $\mathrm{n}=50$ \\
$10,0 \%$
\end{tabular}

Gesamt: $n=500$

Darauf aufbauend ergeben sich durch die Conjoint-Analyse folgende Werte für die Teilnutzenwerte der Eigenschaftsausprägungen:

Tabelle 2: Teilnutzen der Eigenschaftsausprägungen bei PCs für Konsumenten

\begin{tabular}{|l|l|r|r|}
\hline Eigenschaft & Eigenschaftsausprägungen & Teilnutzen & Rang \\
\hline+ Leistung & 1 Prozessor mit 3 GHz & $-1,414$ & 12. \\
& 2 Prozessoren mit je 3 GHz & $-0,004$ & 7. \\
& 4 Prozessoren mit je 3 GHz & 1,418 & 1. \\
\hline+ Preis & 400 Euro & 0,587 & 4. \\
& 500 Euro & 0,005 & 6. \\
& 600 Euro & $-0,592$ & 9. \\
\hline+ Energie & Energieverbrauch von 100 Watt & 0,614 & 3. \\
& Energieverbrauch von 175 Watt & 0,023 & 5. \\
& Energieverbrauch von 250 Watt & $-0,637$ & 11. \\
\hline+ Entsorgung & PC kann nicht recycelt werden & $-0,619$ & 10. \\
& Keine Information über Recycling & $-0,098$ & 8. \\
& PC kann komplett recycelt werden & 0,717 & 2. \\
\hline = Gesamtnutzen & & & \\
\hline
\end{tabular}

Bei den Teilnutzenwerten handelt es sich um absolute Werte. Ein positiver Teilnutzenwert steht für einen Nutzenzuwachs und ein negativer Wert für eine Nutzenminderung. Unter der Bedingung eines additiven Modells werden die Teilnut- 
zen der realen Ausprägungen und der Basisnutzen, der für alle Teilnehmer in diesem Fall 5,000 beträgt, addiert. Den größten Nutzenzuwachs bringt der Prozessor mit der höchsten Leistung. Diese Ausprägung belegt den ersten Rang. Der zweithöchste Nutzenzuwachs wird dadurch erreicht, dass der PC komplett recycelt werden kann.

Um die Qualität der Conjoint-Analyse besser einschätzen zu können, werden die Korrelationen zwischen beobachteten und geschätzten Präferenzen berechnet. Sowohl für die Korrelation nach Pearson als auch für die Rangkorrelation KendallTau ergibt sich ein Wert von 1,000 bei einer Signifikanz von 0,000. Beim KendallTau für die drei Prüffälle (Konzepte zur Prüfung auf Validität) ergibt sich ein Wert von 1,000 bei einer Signifikanz von 0,059. Dies unterstreicht, dass die Ergebnisse der Conjoint-Analyse die beobachteten Werte sehr gut verdichten.

Backhaus et al. (2006, S. 609) empfehlen, die Ergebnisse einer ConjointAnalyse zu klassifizieren. Es ist anzunehmen, dass die Antworten nur selten homogen sind. Zur Klassifizierung wird daher eine Clusteranalayse nach der WardMethode durchgeführt. Zur Distanzbestimmung wird der quadrierte Euklidische Abstand herangezogen (Backhaus et al. 2006, S. 609).

Für eine Clusteranalyse muss die optimale Anzahl an Clustern bestimmt werden. Die Berechnung sollte auf statistischen Kriterien beruhen und nicht aufgrund von sachlogischen Zusammenhängen wie der Einteilung in LOHAS und NichtLOHAS erfolgen (Backhaus et al. 2006, S. 534). Daher wird die Fehlerquadratsumme der Distanzen der Personen untereinander bestimmt. Nach der sogenannten „Elbow"-Methode wird die optimale Anzahl an Clustern ermittelt, die im vorliegenden Fall vier beträgt.

Tabelle 3: Relative Wichtigkeiten der PC-Eigenschaften für die Befragten

\begin{tabular}{|c|c|c|c|c|c|c|}
\hline \multicolumn{2}{|l|}{ Cluster } & Gesamt & $\begin{array}{l}\text { Leistungs- } \\
\text { orientierte }\end{array}$ & $\begin{array}{c}\text { Preisorien } \\
\text { tierte }\end{array}$ & $\begin{array}{l}\text { Energie- } \\
\text { orientierte }\end{array}$ & $\begin{array}{c}\text { Entsor- } \\
\text { gungsorien } \\
\text { tierte }\end{array}$ \\
\hline & aft & $\begin{array}{c}\mathrm{n}=500 \\
100 \%\end{array}$ & $\begin{array}{c}n=228 \\
45,6 \%\end{array}$ & $\begin{array}{c}\mathrm{n}=139 \\
27,8 \%\end{array}$ & $\begin{array}{l}\mathrm{n}=50 \\
10,0 \%\end{array}$ & $\begin{array}{l}\mathrm{n}=83 \\
16,6 \%\end{array}$ \\
\hline Leistung & $\begin{array}{l}\text { Wichtigkeit } \\
\text { SD }\end{array}$ & $\begin{array}{c}37,9 \% \\
22,8\end{array}$ & $\begin{array}{c}59,6 \% \\
12,1\end{array}$ & $\begin{array}{c}22,2 \% \\
9,9\end{array}$ & $\begin{array}{c}20,2 \% \\
10,3\end{array}$ & $\begin{array}{c}15,4 \% \\
9,7\end{array}$ \\
\hline Preis & $\begin{array}{l}\text { Wichtigkeit } \\
\text { SD }\end{array}$ & $\begin{array}{c}21,6 \% \\
17,0\end{array}$ & $\begin{array}{c}13,8 \% \\
8,0\end{array}$ & $\begin{array}{c}42,2 \% \\
17,1\end{array}$ & $\begin{array}{c}14,5 \% \\
7,4\end{array}$ & $\begin{array}{c}12,8 \% \\
7,4\end{array}$ \\
\hline Energie & $\begin{array}{l}\text { Wichtigkeit } \\
\text { SD }\end{array}$ & $\begin{array}{c}19,2 \% \\
14,1\end{array}$ & $\begin{array}{c}14,4 \% \\
9,0\end{array}$ & $\begin{array}{c}17,2 \% \\
10,2\end{array}$ & $\begin{array}{c}49,5 \% \\
11,9\end{array}$ & $\begin{array}{c}17,3 \% \\
10,0\end{array}$ \\
\hline $\begin{array}{l}\text { Entsor- } \\
\text { gung }\end{array}$ & $\begin{array}{l}\text { Wichtigkeit } \\
\text { SD }\end{array}$ & $\begin{array}{c}21,2 \% \\
18,1\end{array}$ & $\begin{array}{c}12,1 \% \\
9,2\end{array}$ & $\begin{array}{c}18,4 \% \\
10,9\end{array}$ & $\begin{array}{c}15,7 \% \\
7,0\end{array}$ & $\begin{array}{c}54,5 \% \\
11,8\end{array}$ \\
\hline
\end{tabular}


Bei vorgegebener Anzahl an Clustern zeigt sich durch die Clusteranalyse, dass jeweils eine Eigenschaft für die Teilnehmer besonders wichtig ist. Die Cluster werden daher als Leistungs-, Preis-, Energie- und Entsorgungsorientierte bezeichnet (vgl. Tabelle 3). Den größten Anteil nehmen die Leistungsorientierten mit 45,6\% ein, gefolgt von den Preisorientierten mit 27,8 \%. Der Anteil der Befragten, für die ökologische Produkteigenschaften eine besondere Rolle bei der Kaufentscheidung spielen, liegt zusammen bei $26,6 \%$. Hiervon stellen die Entsorgungsorientierten $62 \%$ und die Energieorientierten $38 \%$.

Um die Wichtigkeiten der Eigenschaften in Zahlen auszudrücken, werden relative Wichtigkeitswerte bestimmt. Dazu wird wie in Tabelle 3 die Spannweite der Teilnutzenwerte einer Eigenschaft ins Verhältnis zur Summe der Spannweiten aller Eigenschaften gesetzt. Je höher die Wichtigkeit einer Eigenschaft, desto größer ist ihr Einfluss auf die potenzielle Kaufentscheidung eines Konsumenten. Die Summe aller relativen Wichtigkeiten ergibt $100 \%$. Die Wichtigkeit des Energieverbrauchs erreicht 19,2\%. Die Entsorgungseigenschaften liegen mit 21,2\% knapp hinter dem Preis mit 21,6\%. Die Leistung verzeichnet mit einem Anteil von 37,9\% die größte Wichtigkeit. Zusammengefasst nehmen die kumulierten Wichtigkeiten der ökologischen Produkteigenschaften einen Anteil von 40,4\% ein, der damit unter dem Erwartungswert von $50 \%$ bei zwei von vier Eigenschaften liegt. Ökologische Produkteigenschaften können damit als unterdurchschnittlich wichtig bezeichnet werden.

Im weiteren Verlauf ist zu klären, wie sich die mit der Clusteranalyse ermittelten Gruppen in ihren weiteren Merkmalen unterscheiden. Dazu wird eine Diskriminanzanalyse mit der Gruppenzugehörigkeit und neun Variablen, die durch den Fragebogen erhoben wurden, durchgeführt. Tabelle 4 zeigt für die Cluster die fünf Variablen mit der höchsten diskriminatorischen Bedeutung. Die diskriminatorische Bedeutung bewegt sich im Bereich von 0 bis 1. Je höher dieser Wert ist, desto besser trennt das Merkmal die Cluster. Am besten trennt die Intensität, wie oft Dritte (Familie, Freunde oder Bekannte) beim Kauf von PCs beraten werden. Beantwortet wurde die Frage auf einer Skala von 1 für „sehr selten“ bis 5 für „sehr oft“. Insbesondere die Leistungsorientierten beraten Dritte überdurchschnittlich häufig. Beim Merkmal „Geschlecht" ist auffällig, dass die Entsorgungsorientierten im Vergleich zu den anderen Clustern einen deutlich erhöhten Frauenanteil aufweisen. Die Energieorientierten sind überdurchschnittlich gebildet und verzeichnen den höchsten Altersdurchschnitt. Insgesamt werden ökologische Produkteigenschaften eher von älteren Umfrageteilnehmern bevorzugt. Aufgrund der Signifikanz von 0,156 kann dieser Zusammenhang jedoch zufällig entstanden sein.

Für den Absatz von PCs ist die Wahrscheinlichkeit, mit der die Befragten innerhalb der nächsten zwölf Monate einen PC kaufen entscheidend. Hierbei schneiden die ökologischen Produkteigenschaften unterdurchschnittlich ab. Daraus kann geschlussfolgert werden, dass der Markt insbesondere durch die Leistungs- und Preisorientierten geprägt ist. 
Tabelle 4: Charakteristische Merkmale der Cluster

\begin{tabular}{|l|c|c|c|c|c|}
\hline Cluster & $\begin{array}{c}\text { Andere } \\
\text { beraten } \\
\text { (Skala: } 1 \text { bis } \\
5)\end{array}$ & $\begin{array}{c}\text { Geschlecht } \\
\text { Frauen / Männer }\end{array}$ & $\begin{array}{c}\text { Bildung } \\
\text { (Anteil der } \\
\text { Akademiker })\end{array}$ & $\begin{array}{c}\varnothing \text { Alter } \\
\text { in Jahren }\end{array}$ & $\begin{array}{c}\text { Neuer PC } \\
\text { im } \\
\text { nächsten } \\
\text { Jahr }\end{array}$ \\
\hline Durchschnitt & 3,2 & $19,8 \% / 80,2 \%$ & $15,2 \%$ & 26,6 & $32,2 \%$ \\
\hline $\begin{array}{l}\text { Leistungsorien- } \\
\text { tierte }\end{array}$ & 3,5 & $15,8 \% / 84,2 \%$ & $14,9 \%$ & 25,7 & $36,1 \%$ \\
\hline Preisorientierte & 2,8 & $18,0 \% / 82,0 \%$ & $10,8 \%$ & 26,6 & $31,4 \%$ \\
\hline $\begin{array}{l}\text { Energieorien- } \\
\text { tierte }\end{array}$ & 3,1 & $20,0 \% / 80,0 \%$ & $30,0 \%$ & 29,3 & $28,0 \%$ \\
\hline $\begin{array}{l}\text { Entsorgungs- } \\
\text { orientierte }\end{array}$ & 2,9 & $33,7 \% / 66,3 \%$ & $14,5 \%$ & 27,2 & $25,4 \%$ \\
\hline $\begin{array}{l}\text { Diskriminatoris } \\
\text { che Bedeutung }\end{array}$ & 0,537 & 0,296 & 0,286 & 0,233 & 0,233 \\
\hline $\begin{array}{l}\text { Signifikanz bei } \\
\text { einem Gleich- } \\
\text { heitstest der } \\
\text { Gruppenmit- } \\
\text { telwerte }\end{array}$ & 0,000 & 0,005 & 0,046 & 0,156 & 0,000 \\
\hline
\end{tabular}

Die Ergebnisse zeigen, dass das komplette Recycling eines PCs sowie ein niedriger Energieverbrauch einen großen positiven Nutzen für die Befragten stiften. Für 16,6 \% der Befragten ist die Entsorgung des PCs das wichtigste Entscheidungskriterium ist. Für $10 \%$ der Befragten ist der Energieverbrauch ausschlaggebend für die Auswahl eines PCs. Daraus folgt, dass ökologische Produkteigenschaften einen Einfluss auf die Kaufentscheidung ausüben. Die Wichtigkeit liegt jedoch mit einem Anteil von 40,4\% an der Kaufentscheidung unter dem Erwartungswert und kann damit als unterdurchschnittlich bezeichnet werden.

Die Vermutung, dass der Marktanteil für PCs mit ökologischen Produkteigenschaften mindestens ein Drittel am Gesamtmarkt für PCs ausmacht, kann nicht bestätigt werden. Werden die Energie- und Entsorgungsorientierten zusammengefasst, ergibt sich ein geschätzter Marktanteil von 26,6\%. Dieser liegt knapp unterhalb des Anteils der LOHAS. Daraus kann geschlussfolgert werden, dass der Trend zu umweltorientierten Produkten den Markt für IT-Hardware noch nicht so stark erreicht hat, wie es zum Beispiel in der Lebensmittelbranche der Fall ist.

Dennoch ist es anhand der gewonnen Ergebnisse möglich, den Markt für PCs besser zu verstehen und konkrete Handlungsempfehlungen unter dem Aspekt der Green IT abzuleiten. 


\section{$5 \quad$ Handlungsempfehlungen}

Aus den Ergebnissen lassen sich Handlungsempfehlungen für das MarketingProgramm und die darin enthaltenen absatzpolitischen Instrumente der ITHersteller ableiten. Zu diesen Instrumenten gehören die Produktpolitik, die Preispolitik, die Kommunikationspolitik und die Distributionspolitik (Scharf und Schubert 2001, S. 55-56).

Die Produktpolitik befasst sich mit der Ausgestaltung des Absatzprogramms und der Entwicklung sowie der Verbesserung von Produkten (Scharf und Schubert 2001, S. 55). Die Ergebnisse zeigen, dass ökologische Produkteigenschaften bei der Kaufentscheidung eine Rolle spielen. Die Leistung und der Preis bleiben die dominierenden Faktoren bei der Entscheidung für oder gegen einen PC. Dennoch sind ein niedriger Energieverbrauch und Angaben zur verantwortungsvollen Entsorgung weitere wichtige Kaufargumente. Dieses muss bei der Produktentwicklung und -verbesserung berücksichtigt werden. Die höhere Bedeutung der Entsorgungseigenschaften (62\%) gegenüber den Energieeigenschaften (38\%) kann beispielsweise im Rahmen der Zielkostenrechnung genutzt werden. Für das Absatzprogramm empfiehlt sich eine Erweiterung der Produktpalette um umweltorientierte Produkte, um den Wünschen der Energie- und Entsorgungsorientierten zu entsprechen.

Die Preispolitik beinhaltet alle Maßnahmen zur ziel- und marktgerechten Gestaltung des Preises (Scharf und Schubert 2001, S. 55). Die Untersuchung ergab, dass es für PCs mit ökologischen Produkteigenschaften eine höhere Preisbereitschaft gibt. Akademiker, die tendenziell über ein höheres Einkommen verfügen sind weniger preisorientiert und mehr energieorientiert. Daraus leitet sich die Handlungsempfehlung ab, einen PC mit ökologischen Produkteigenschaften im oberen Preissegment zu platzieren.

Die Kommunikationspolitik umfasst sämtliche Maßahmen, die darauf abzielen, die Kenntnisse, Einstellungen und Verhaltensweisen von Marktteilnehmern zu beeinflussen (Scharf und Schubert 2001, S. 56). Die Kommunikationsstrategie sollte den erhöhten Anteil von weiblichen Interessenten an ökologischen Produkteigenschaften berücksichtigen. Außerdem ist die relevante Zielgruppe tendenziell älter und besser gebildet.

Die Distributionspolitik bezieht sich auf alle Entscheidungen, die den Weg des Produktes zum Endkäufer betreffen (Scharf und Schubert 2001, S. 56). Insbesondere Entsorgungsorientierte beraten im Gegensatz zu den Leistungsorientierten andere nur unterdurchschnittlich häufig. Hierbei lässt sich ein geringerer Wissensstand über PCs vermuten. Der Vertrieb von PCs mit ökologischen Produkteigenschaften sollte daher durch persönliche Beratung unterstützt werden.

Mit diesen Maßnahmen lässt sich ein Marksegment von bis zu 26,6 \% der potenziellen Konsumenten von PCs mit ökologischen Produkteigenschaften erschließen. 
Desweiteren geben die Ergebnisse Hinweise für IT-Organisationen in Unternehmen, die PCs für den Arbeitsplatz bereitstellen und eine zunehmende Kundenorientierung betreiben. So ist davon auszugehen, dass Unternehmensmitarbeiter ökologische Produkteigenschaften ebenfalls eine gewisse Bedeutung beimessen. Die Berücksichtigung dieser Wünsche steigert damit tendenziell die Zufriedenheit der Mitarbeiter mit der IT-Organisation.

\section{$6 \quad$ Fazit}

Im Rahmen dieser Arbeit wurde die Bedeutung von ökologischen Produkteigenschaften bei PCs auf das Konsumentenverhalten aufgezeigt. Die Conjoint-Analyse mit 500 Teilnehmern verdeutlicht, dass die Leistung das dominierende Kriterium beim Kauf von PCs ist. Jedoch hat Green IT, in Form von Energie- und Entsorgungseigenschaften, einen Einfluss auf die potenzielle Kaufentscheidung und liegt dicht unterhalb der Bedeutung des Preises. Darüber hinaus konnte gezeigt werden, dass für ca. ein Viertel der Befragten eine der ökologischen Produkteigenschaften das dominierende Entscheidungsmerkmal ist.

Die abgeleiteten Handlungsempfehlungen bieten IT-Herstellern und CIOs neue Ansätze Green IT im Bereich der PCs gewinnbringend und imagefördernd zu vermarkten. Vor dem Hintergrund einer wachsenden Bedeutung ökologischer Themen in der IT werden IT-Herstellern und IT-Organisationen hiermit Ansätze aufgezeigt, sich zukunftsfähig zu positionieren.

Weiterer Forschungsbedarf besteht insbesondere darin die Ergebnisse durch ähnliche Untersuchung zu validieren. Darüber hinaus ist zu erwarten, dass sich die Einstellung der Befragten zu Green IT im deutschsprachigen Raum im Vergleich zu anderen Märkten unterscheidet. Eine Übertragbarkeit der Ergebnisse auf andere Märkte ist somit nicht gegeben. Schließlich bleibt die Frage, inwieweit die Ergebnisse auf interne Märkte übertragbar sind. Hierzu ist eine Befragung von Mitarbeitern in Unternehmen vorgesehen, die ihre PCs von einer IT-Organisation zur Verfügung gestellt bekommen.

\section{Literatur}

Albers S (1984) Fully nonmetric estimation of a continuous nonlinear conjoint utility function. In: International Journal of Research in Marketing 1(4):311319.

Apple Inc. (2009) 13-inch MacBook Pro. http://images.apple.com/environment/resources/pdf/MacBook-Pro-13-inchEnvironmental-Report.pdf. Abruf am 2009-08-01.

Backhaus K, Erichson B, Plinke W, Weiber R (2006) Multivariate Analysemethoden - Eine anwendungsorientierte Einführung. Springer, Berlin. 
BITKOM (Bundesverband Informationswirtschaft, Telekommunikation und neue Medien e.V.) (2009a) Verbraucher achten zunehmend auf Energie- und Umwelteigenschaften. http://www.bitkom.org/51996_51978.aspx. Abruf am 2009-05-15.

BITKOM (Bundesverband Informationswirtschaft, Telekommunikation und neue Medien e.V.) (2009b) Green-IT. http://www.bitkom.org/de/themen/51049.aspx. Abruf am 2009-09-12.

Dellaert B, Borgers A, Timmermans H (1996) Conjoint choice models of joint participation and activity choice. In: International Journal of Research in Marketing 13(3):251-264.

Diller H (2008) Preispolitik. Kohlhammer, Stuttgart.

Dellaert B, Borgers A, Timmermans H (1996) Conjoint choice models of joint participation and activity choice. In: International Journal of Research in Marketing 13(3):251-264.

Du S, Bhattarchaya CB, Sen S (2007) Reaping relational rewards from corporate social responsibility: The role of competitive positioning. In: International Journal of Research in Marketing 24(3):224-241.

Foscht T, Swoboda B (2007) Käuferverhalten: Grundlagen - Perspektiven Anwendungen. Gabler, Wiesbaden.

Görts T, Behringer T (2003) Online Conjoint - Chancen und Grenzen: Ein Fallbeispiel aus dem Telekommunikationsmarkt. In: Theobald A, Dreyer M, Starsetzki T (Hrsg) Online-Marktforschung - Theoretische Grundlagen und praktische Erfahrungen. Gabler, Wiesbaden.

Heidbrink M (2006) Reliabilität und Validität von Verfahren der Präferenzmessung - Ein meta-analytischer Vergleich verschiedener Verfahren der ConjointAnalyse. Dissertation, Münster.

Heng S (2009) Green-IT: IT is not green and never ever will be!. http://www.dbresearch.de/PROD/DBR_INTERNET_DEPROD/PROD0000000000238000.pdf. Abruf am 2009-05-26.

Lichtenstein DR, Drumwright ME, Braig BM (2004) The effect of Corporate Social Responsibility on Customer Donations to Corporate-Supported Nonprofits. In: Journal of Marketing 68(4):16-32.

Luo X, Bhattacharya CB (2006) Corporate Social Responsibility, Customer Satisfaction, and Market Value. In: Journal of Marketing 70(4):1-18.

Meffert H (2000) Marketing - Grundlagen marktorientierter Unternehmensführung. Gabler, Wiesbaden.

Müller-Hagedorn L (2005) Handelsmarketing. Kohlhammer, Stuttgart. 
Scharf A, Schubert B (2001) Marketing - Einführung in Theorie und Praxis. Schäffer-Poeschel, Stuttgart.

Schmidt NH, Erek K, Kolbe LM, Zarnekow R (2009) Towards a Procedural Model for Sustainable Information Systems Management. In: Sprague RH (Hrsg) Proceedings of the 42th Hawaii International Conference on System Sciences 2009 (HICSS-42), Hawaii.

Wenzel E, Kirig A, Rauch C (2007) Zielgruppe LOHAS - Wie der grüne Lifestyle die Märkte erobert. Zukunftsinstitut, Kelkheim.

Wittink DR, Vriens M, Burhenne W (1994) Commercial use of conjoint analysis in Europe: Results and critical reflections. In: International Journal of Research in Marketing 11(1):41-52.

Wyner GA (1992) Uses and Limitations of Conjoint Analysis - Part 1. Marketing Research 4(2):42-44.

Zerr K (2003) Online-Marktforschung - Erscheinungsformen und Nutzenpotenziale. In: Theobald A, Dreyer M, Starsetzki T (Hrsg) OnlineMarktforschung - Theoretische Grundlagen und praktische Erfahrungen. Gabler, Wiesbaden. 